\title{
¿Una pareja dispareja? Prebisch, Keynes y la dinámica capitalista
}

\author{
Esteban Pérez Caldentey \\ Matías Vernengo
}

Resumen. Keynes tuvo una profunda influencia en el pensamiento de Prebisch, no sólo en términos del diagnóstico acerca de los principales fallos de las economías de mercado, sino también en la necesidad de llevar a cabo políticas proactivas y anticíclicas. No obstante, Prebisch fue crítico de la obra principal de Keynes, Teoría general de la ocupación, el interés y el dinero (1936). Consideró que dicha obra estaba alejada de la realidad de las economías capitalistas. Además sostuvo que era inconsistente y no representaba en absoluto una ruptura con la teoría dominante. Las críticas de Prebisch se centraron en la teoría de interés y el multiplicador. La actitud de Prebisch en relación a Keynes puede explicarse por una diferencia en el objeto y el método de análisis. Los intereses de Prebisch se centraban en la dinámica y los ciclos, temas periféricos al mensaje y análisis central de Teoría general. Sin embargo, hay muchos puntos en común y confluencias entre ambos autores.

Palabras Clave: Prebisch, Keynes, clásicos, tasa de interés, multiplicador.

Abstract. Keynes had a profound influence on Prebisch, not only in terms of his diagnosis of the main failures of market economies, but also on the need to pursue pro-active and anti-cyclical policies. However, Prebisch was critical of Keynes' most important publication, The General Theory of Employment, Interest and Money (1936). He viewed this work as being removed from the reality of capitalist economies. He also argued that it was inconsistent and did not represent at all a break with conventional wisdom. Prebisch's criticisms focused on the theory of interest and the multiplier. Prebisch's attitude in relation to Keynes can be explained by a difference in the object and method of analysis. The former's interests focused on dynamics and cycles, themes that were peripheral to the central message and analysis of The General Theory. Notwithstanding Prebisch's criticisms, there are several similarities between his analysis and that of Keynes.

Keywords: Raúl Prebisch, Keynes, classics, rate of interest, multiplier.

Esteban Pérez Caldentey trabaja en la Comisión Económica para América Latina (CEPAL) y Matías Vernengo trabaja en el Banco Central de Argentina (BCRA) y University of Utah. Las opiniones aquí expresadas son de exclusiva responsabilidad de los autores y pueden no coincidir con las de la CEPAL o el BCRA. 


\section{INTRODUCCIÓN}

ohn Maynard Keynes ejerció una profunda influencia en el pensamiento de Raúl Prebisch. Esta influencia fue notoria en el progresivo cuestionamiento que hizo Prebisch a algunas de las recomendaciones de política económica que emanaban de la teoría neoclásica o más ortodoxa de la época. ${ }^{1}$ Además, Prebisch compartía el diagnóstico de Keynes acerca de los fallos esenciales de las economías de mercado, a saber, la imposibilidad para procurar el pleno empleo y la arbitraria distribución del ingreso y la riqueza. También concordaba con las propuestas de política económica keynesianas, en particular en lo que respecta al activismo en política económica, ya sea en el plano monetario, fiscal o internacional.

No obstante, Prebisch fue un crítico del magnum opus de Keynes, Teoría general de la ocupación, el interés y el dinero (1936) (TG). ${ }^{2}$ En particular, criticó la falta de consistencia, la similitud con la teoría clásica y el alejamiento de la realidad. Así, con respecto a los dos primeros aspectos, Prebisch señaló que sus inconsistencias eran tales que no se podía emplear la Teoría general «como instrumento para explorar la realidad, por adolecer de los mismos graves defectos [que la teoría neoclásica]» (Prebisch, 1993, vol. IVะ 278). ${ }^{3}$ Prebisch centró su ataque en la teoría del interés de Keynes y en su análisis del multiplicador. Esto pese a haber desarrollado por cuenta propia una versión del multiplicador de comercio exterior, al cual denominó el coeficiente de expansión.

\footnotetext{
${ }^{/ 1 /}$ Los paréntesis en las citas, frases en negritas y las traducciones son de los autores de este artículo. Los cuatro volúmenes de las obras de Prebisch aparecen citados por Prebisch, 1991, volumen I a iII y por Prebisch, 1993, volumen IV.

12/ Las referencias a la Teoría general de Keynes son a su edición en inglés (1936 [1964]).

${ }^{13 /}$ Cabe clarificar que, como Keynes, Prebisch se refiere a la teoría dominante de la época como clásica y no hace distinción con los autores de la vieja economía política o el abordaje del excedente (i.e., Smith, Ricardo y, en menor medida, Marx). Al contrario de ambos, aquí mantendremos la distinción fundamental entre clásicos y neoclásicos.
} 
En términos de su relevancia, la crítica de Prebisch fue aún más enfática: «[...] el keynesianismo se ha convertido en un dogma que sus discípulos ya no discuten, sino que lo toman como la verdad revelada [...] y exigen sobre el conjunto de las llamadas verdades keynesianas nuevas construcciones teóricas que se alejan cada vez más de la realidad, como es a mi juicio, la misma construcción de Keynes» (Prebisch, 1991, vol. iII: 504).

La actitud de Prebisch hacia la Teoría general se explica por una diferencia en el objeto y método de análisis. Desde los inicios de su carrera profesional, el interés fundamental de Prebisch se centró en el estudio del ciclo. Prebisch pensaba que la realidad de las economías de mercado era eminentemente cíclica. El ciclo era la típica forma de crecer del capitalismo. Esto no sólo significaba que la teoría económica debía centrarse en las causas, la intensidad y, quizá, la duración de las fases del ciclo, sino que debía ser esencialmente una teoría dinámica. Esta investigación fue orientándose en esta dirección hasta culminar en una serie de clases y escritos sobre la «Teoría dinámica de la economía» (Vernengo y Pérez Caldentey, 2012b).

En cambio, los esfuerzos de Keynes se orientaron en otra dirección. El tema central de la Teoría general es la explicación del nivel de empleo, producto y uso de la capacidad ya existentes. Y el ciclo y la dinámica son de hecho temas periféricos. ${ }^{4}$ Los temas dinámicos y de ciclos en base a la teoría de Keynes fueron desarrollados por otros autores, incluyendo, entre los más conocidos, a Roy Harrod y Joan Robinson. ${ }^{5}$ Pese a su desilusión por la teoría ortodoxa y

/4/ Amadeo (1989) argumenta correctamente que en la transición de la Teoría general al Tratado de la moneda, Keynes se movió de una teoría cíclica del funcionamiento de la economía, pero convencional y todavía prisionera, de la Ley de Say, a una teoría de equilibrio, aunque con la incorporación del principio de la demanda efectiva.

15/ En particular, Harrod y Robinson intentaron dinamizar la teoría keynesiana y extender la demanda efectiva al largo plazo. Sobre los límites de esos intentos, ver Vernengo y Rochon (2001). Otros dos autores esenciales en la tradición keynesiana fueron Kalecki y Kaldor. Todos estos autores, así como Prebisch, enfatizaban la dinámica cíclica del proceso de crecimiento e intentaban mostrar que el pro- 
a su esfuerzo por hacer asequible la Teoría general, en la Introducción a Keynes (1947) Prebisch jamás encontró lo que buscaba. Esto quizá explica la razón por la cual, al desarrollar su «Teoría dinámica», otorgó un papel central a un concepto clave para la teoría neoclásica, el ahorro forzoso.

El resto de este artículo se divide en cuatro secciones y una breve conclusión. La primera sección examina los primeros contactos de Prebisch con la obra de Keynes, discute su Introducción a Keynes (1947) y describe la disconformidad de Prebisch con la teoría económica incluyendo la del propio Keynes. La segunda y tercera secciones analizan las críticas de Prebisch a la Teoría general, la primera a su teoría de la tasa de interés y la subsecuente a la teoría del multiplicador. La tercera sección trata de la evaluación de Prebisch de los planes monetarios internacionales, incluido La Unión de Compensaciones de Keynes, que sirvió de base para la postura británica en las negociaciones de Bretton Woods, y brevemente identifica diferencias en las perspectivas históricas del análisis de Prebisch y Keynes. La conclusión evalúa críticamente la relación intelectual entre las obras de Keynes y Prebisch.

\section{Leyendo a Keynes en Buenos Aires}

Prebisch inició su carrera de economista a principio de la década de los años veinte, centrándose en el estudio de las fluctuaciones del ciclo económico y su relación con el dinero y las finanzas. En gran medida, este interés surgió a raíz de su estudio de la historia económica argentina y se consolidó por su experiencia práctica posterior en el Ministerio de Finanzas y en el Banco Central. Prebisch creía que los ciclos eran hechos «naturales», «recurrentes»

ceso dinámico no era de equilibrio, por lo menos en el sentido de que las fluctuaciones no se daban alrededor de un centro de gravitación óptimo. Besomi (2002) sugiere que Harrod, como Prebisch, con su ensayo sobre la dinámica, intentaba algo más allá que extender la teoría keynesiana: revolucionar la teorización en economía. 
e inevitables de la vida económica y que se caracterizaban por fases de auge y declive. Además, en línea con Juglar, pensaba que las distintas fases estaban relacionadas (la dureza de la fase contractiva tenía una relación directa con los excesos de la fase expansiva). De manera más precisa, la profundidad de la fase contractiva tenía una relación directa con la intensidad de la fase de auge: cuanto más grandes fueran los excesos del auge, más drástico sería el colapso. Al mismo tiempo, el colapso no sólo era inevitable, sino que era necesario para prepararse para la próxima fase ascendente (Prebisch, 1991, vol. I: 618 y 634).

Prebisch pensaba que los intentos por impedir la fase contractiva del ciclo (y más específicamente, un colapso) sólo podían tener efectos temporales y que, en última instancia, resultaban inútiles en un proceso que era necesario para restaurar el equilibrio externo (una condición sine qua non para lograr el equilibrio interno). Además, al posponer lo que se considera un proceso natural, estas medidas a la larga se convierten en artificios que tienden a agravar las fuerzas correctivas necesarias.

Al igual que algunos de los más destacados economistas de la época, como Schumpeter, en un principio Prebisch creía en la teoría de la liquidación y evaluó los efectos del colapso como naturales y saludables, considerándoles una especie de limpieza de elementos nocivos (Prebisch, 1991, vol. iI: 601; vol. I: 171). Sin embargo, a fines de la década de los veinte, influenciado por $E l$ tratado sobre el dinero (1923) de John Maynard Keynes y por otros economistas, Prebisch finalmente reconoció los desagradables y prolongados efectos del ajuste y la deflación en la actividad económica. ${ }^{6}$ En este aspecto, es útil citar a Prebisch in extenso (1991, vol. II* 47-48):

16/ Otro autor que puso énfasis en los efectos negativos de la deflación fue Silvio Gesell (1862-1930), un economista alemán que vivió en Argentina, entre 1886 y 1900, y que —al referirse al periodo específico de fines del siglo xix en Argentina- dijo que el aumento del valor del dinero era la causa de todos los problemas económicos del país (Gesell, 1898). Estos efectos también fueron destacados en los inicios de la Escuela de Economía de Chicago en términos similares a los utilizados por Prebisch en esta etapa de su pensamiento (es decir, en términos de precio nominal y rigideces de los salarios) y 
Una teoría económica muy difundida, de linaje ortodoxo, enseña, en efecto, que situaciones de esa índole, en tiempos de crisis se resuelven por la liquidación. La teoría es perfectamente exacta dentro de las hipótesis sobre la que se basa. Supone que el caso de la producción que gracias a la ayuda del crédito se ha exagerado más allá de la capacidad de consumo del mercado, o el de la valoración ficticia de inmuebles [...] fomentada especulativamente por la abundancia de crédito. Tarde o temprano el mercado no puede absorber estas mercaderías o valores a los precios vigentes o esperados. La venta se paraliza y los productores no pueden cumplir con sus compromisos bancarios. La misma teoría receta el remedio: restrínjase el crédito y oblíguese a liquidar [...] a ofrecer esas mercaderías a precios más bajos [...] Con la liquidación [...] la situación se despeja, a costa de pérdidas y quiebras, pero el mal desaparece. Los ineptos son desalojados y sobreviven por selección los mejor dotados [...] La más leve reflexión basta para persuadirse de que esa teoría de la liquidación corresponde a datos y hechos muy distintos a los de la realidad argentina.

Probablemente sobre la base de la lectura del capítulo primero del Tratado que examina, entre otros aspectos, las consecuencias reales y sociales de los cambios en el valor del dinero, Prebisch reconoció la existencia de rigideces en la forma de organización de las economías de libre mercado que impedía el ajuste requerido por la teoría de la liquidación para sanar y restaurar la plena salud de la economía. Quizá Prebisch también fue influenciado por Las consecuencias económicas del Sr. Churchill (1925) y otros ensayos de esos años que abordan temas similares (Keynes, 1932). 
De manera más precisa, Prebisch recalcó que los contratos se establecen en términos de dinero, y que los costos como los salarios y, en general, los costos de producción son rígidos a la baja. Además, la deflación no incentivaba la expansión del empleo y del producto porque aumentaba también la carga de la deuda (Prebisch, 1991, vol. I: 59-60 y 135). Estos argumentos fueron utilizados por Prebisch y sentaron las bases para cuestionar los beneficios de la liquidación durante la fase descendente del ciclo. ${ }^{7} \mathrm{Al}$ cuestionar los efectos benéficos de la liquidación, Prebisch también cuestionó, como corolario y secuencia lógica, la utilidad y los efectos positivos de la prociclicidad en la política económica, que incluso él mismo había propugnado en varias ocasiones (Prebisch, 1993, vol. IV 116-117). ${ }^{8}$

Los primeros intentos de Prebisch para aplicar medidas de política contracíclicas se remontan a 1933. Según Prebisch, en esta etapa Argentina efectuó los primeros intentos, aunque tímidos y temporales, para implementar políticas contracíclicas consistentes en sostener el precio de los productos agrícolas mediante compras estatales y la realización de obras públicas. No hay duda de que la influencia de la obra Los medios para la prosperidad de Keynes, que Prebisch había leído en ese año, fue fundamental en el diseño de estas medidas (Prebisch, 1991, vol. 2: 146). A medida que esta visión se fue consolidando, el abanico de políticas contracíclicas se fue ampliando. Un

17/ Véase también La inflación escolástica y la moneda argentina (Prebisch, 1991, volúmenes II y III: 336350). La liquidación era una de las fases del ciclo identificadas por Juglar, que se relacionó con la teoría austríaca del ciclo económico (véase, por ejemplo, Schumpeter, 1989; Hayek, 1933) y con políticas pasivas adoptadas por la Reserva Federal y la administración de Herbert Hoover que profundizaron la Gran Depresión (puede verse una opinión contraria en White, 2010). Eichengreen (1999: 8 y 12) habla de «liquidacionismo», un concepto según el cual los descensos del ciclo económico cumplían con la función darwiniana de eliminar a las empresas más débiles, que estaban menos adaptadas a una economía dinámica. Como puede observarse, Prebisch comprendía la liquidación y sus efectos en un sentido muy contemporáneo, y que aún sigue vigente en círculos neoclásicos.

${ }^{18 /}$ Cuando era subsecretario de Hacienda, Prebisch puso en práctica lo que denominó «ajustes brutales del presupuesto», entre los que se incluía una reducción de $15 \%$ en los salarios públicos (véase Pollock, Kerner y Love (2002: 543). 
primer paso en esta dirección fue el abandono del patrón oro y la utilización de medidas discrecionales, como el redescuento (pese a que no tuvo éxito) y los controles de cambios que sentaron las bases para la creación del Banco Central, con Prebisch a la cabeza, y la adopción de una política monetaria autónoma y proactiva. ${ }^{9}$

Esto se materializó en garantizar un nivel adecuado de acumulación de reservas por motivo de precaución para enfrentar y amortiguar los choques de exportaciones y las detenciones bruscas de capital. De manera más general, Prebisch articuló la necesidad de una política monetaria de la siguiente manera (Prebisch, 1991, vol. niı: 104):

[...] la política monetaria puede proponerse dos objetivos frente al desarrollo del ciclo económico. El primero consiste en evitar que la expansión del crédito acentúe la intensidad de los movimientos ondulatorios [...] El segundo objetivo va más lejos. No se limita a evitar que estas fluctuaciones se acentúen, sino que se propone, además, reducir su amplitud y disminuir la intensidad con que varía la masa del poder adquisitivo durante el proceso cíclico, para atenuar las consecuencias de tales variaciones sobre el volumen de la actividad económica interna.

La necesidad de adoptar medidas contracíclicas volvió a resurgir temporalmente poco después del inicio de la Segunda Guerra Mundial, con el Plan para la Reactivación Nacional, y en 1942 cuando Argentina debió enfrentar

19/ Prebisch afirma, no obstante, que había percibido la necesidad de crear una autoridad monetaria central antes de la Primera Guerra Mundial (Prebisch, 1991, vol. I: 7). En su entrevista con Prebisch en 1983, Julio González del Solar (Mallorquín, 2006) consideró que el uso del redescuento y la creación de la Comisión para el Control de Divisas en 1931 fueron los primeros dos pasos heterodoxos del pensamiento de Prebisch. Sin embargo, Prebisch consideraba al redescuento un instrumento ortodoxo. Por otra parte, él creía que los controles de cambios en cierto modo se apartaban de la doctrina central (Prebisch, 1991, vol. III: 89). 
un creciente desequilibrio externo y cuando se percibía como inminente una contracción económica. También se incluyó el uso breve de controles de capital en 1943, mucho antes que fueran propuestos por Keynes y White en Bretton Woods (1944). Finalmente, en su libro inconcluso La moneda y el ritmo de la actividad económica, en el que venía trabajando desde 1943, Prebisch otorga amplias funciones a la política monetaria; afirma que la política monetaria y financiera deberían tener tres objetivos centrales: $a$ ) atenuar los efectos de los cambios abruptos en las condiciones de la cosecha, las fluctuaciones y las contingencias externas; $b$ ) crear condiciones monetarias que estimulen el desarrollo y el mantenimiento del pleno empleo de la fuerza laboral, y c) promover y apoyar la mayor tasa de crecimiento posible de la actividad económica.

Éstas encontrarán eco en las reformas realizadas por la Reserva Federal de Estados Unidos en diversos países latinoamericanos bajo la dirección de Robert Triffin (Vernengo y Pérez Caldentey, 2012a). Después de su abrupta salida del Banco Central de la República Argentina, Prebisch dedicó más tiempo no solamente a sus actividades como consultor económico, sino también a la enseñanza. Su discusión de la Teoría general se profundiza en ese periodo.

La Introducción a Keynes fue la culminación de una serie de artículos publicados en el Boletín del Banco Central de Venezuela (enero-febrero y abril-mayo de 1947, núms. 23-24 y 26-27). Prebisch publicó su Introducción a Keynes en 1947, seis años antes que Hansen publicara su Guía de Keynes. El propósito de Prebisch quedó claramente establecido en el prólogo a esta obra. Prebisch no buscaba otra cosa más que una explicación del ciclo económico y una teoría de la dinámica en Keynes, una explicación alternativa a la teoría económica convencional y más acorde a la realidad. Tal y como explicó (Prebisch, 1947: 13):

Este desempeño mío en exponer el pensamiento keynesiano responde a una preocupación muy justificada en quién ha tenido alguna responsabilidad en la política monetaria argentina de años pasados: la de encontrar en la teoría la explicación racional de los movimientos de la realidad 
económica, a fin de actuar inteligentemente sobre ella. La teoría de esos movimientos, la teoría dinámica de la economía, ha comenzado a desarrollarse en las últimas décadas. Le hacía falta un impulso vigoroso. Keynes ha tenido una clara noción de ello: de ahí la oportunidad y trascendencia de su obra. ${ }^{10}$

La intención de Prebisch era simplemente de exponer y resumir la teoría de Keynes. No obstante, Prebisch se planteó analizar el trabajo y obra de Keynes y evaluar si efectivamente había podido «dar una explicación racional del movimiento económico y dilucidar los principios a los cuales éste obedece». En sus palabrasः «es lo que me propongo considerar en un trabajo de más aliento sobre el sistema keynesiano» (ibid.: 14).

La Introducción a Keynes consta de cinco partes. La primera parte («Introducción al Sistema Keynesiano») cubre los libros I y ir de la Teoría general («Introducción» y «Definición de Ideas»). La segunda parte («La Propensión a Consumir y la Teoría del Multiplicador») se centra en el libro in de Keynes («La propensión a consumir»). La tercera parte («La Eficiencia del Capital y la Tasa de Interés») incluye el libro Iv («El Incentivo para Invertir») desde el capítulo 11 al 14. La parte cuatro («La Conjunción del Ahorro con las Inversiones») se refriere en parte al libro iv (capítulos 13, 17) y al libro vi ( «Notas Breves Sugeridas por la Teoría General», capítulos 22, 23 y 24). La quinta parte («Significado y Proyecciones Sociales del Esquema Keynesiano») se focaliza en el libro IV (capítulo 18), en el libro v (capítulos 19 y 21) y en el libro vi (capítulo 24).

Prebisch no encontraría en Keynes lo que buscaba. El objetivo de la teoría de Keynes (centraba el caso de una economía capitalista avanzada) era

/10/ La traducción al castellano de la Teoría general fue publicada por el Fondo de Cultura Económica en 1943. La segunda edición apareció en 1945, dos años antes que Prebisch publicara su Introducción a Keynes. Prebsich, no obstante, utilizó la edición en inglés para dar mayor precisión al pensamiento de Keynes. Para una crítica de la traducción al castellano de la obra de Keynes en el Fondo de Cultura Económica, véase Braun (1986). 
explicar las causas del aumento o disminución del empleo y la utilización de la capacidad ya existente. $\mathrm{Ni}$ el análisis del ciclo ni la dinámica constituían una preocupación central de la obra de Keynes, siendo la principal preocupación la demostración de la posibilidad de una posición de equilibrio con desempleo. En otras palabras, se trataba de demostrar que las economías de mercado tienden hacia posiciones de equilibrio con desempleo, incluso si se cumplieran todas las condiciones de una situación de competencia perfecta con plena flexibilidad de precios. La razón esencial para explicar la persistencia del desempleo radicaba en que las economías de mercado son, ante todo, economías monetarias, economías en las cuales el dinero juega un papel esencial, porque es el nexo entre el presente y un futuro incierto. ${ }^{11}$

El examen del ciclo tiene un papel secundario en la Teoría general y se concentra esencialmente en el capítulo 22 («Notas sobre el Ciclo Económico»). Keynes sostiene que el ciclo es un fenómeno complejo que involucra en su explicación los distintos elementos de su teoría. El ciclo se explica por las fluctuaciones de la tasa de inversión como consecuencia de variaciones en la eficiencia marginal de capital. Ésta, a su vez, viene determinada por los cambios en los retornos esperados (i.e., cambios en las expectativas) de los nuevos bienes de capital. Las fluctuaciones del ciclo se ven agravadas por otros factores $y$, en particular, por las variaciones en la preferencia por la liquidez. ${ }^{12}$

/11/ Tal y como se expondrá más adelante, el desempleo tiene su explicación en la existencia del dinero o, más precisamente, de una tasa de interés convencional de largo plazo que no permite la plena utilización de los recursos productivos. O sea, Keynes (1936: 242-44) sugería que la tasa natural de interés que garantizaría la plena ocupación y la estabilidad de precios era una quimera teórica y debería ser abandonada.

${ }^{\prime 12 /}$ Así, el colapso de la eficiencia marginal del capital (el reflejo más patente de una crisis) trae consigo un aumento en la preferencia por la liquidez, aumentando así la tasa de interés. Esto es lo que puede hacer tan intratable una contracción o más bien depresión económica (Keynes, 1936: 316). Cabe notar que la eficiencia marginal del capital y de la preferencia por la liquidez, conceptos marginalistas, son infiltrados en la teoría keynesiana. Sobre la necesidad de abandonar los conceptos marginalistas para obtener los resultados keynesianos, véase Camara Neto y Vernengo (2012). 
Hay que añadir, además, que parte del capítulo 22 está más bien dedicado a las crisis que al ciclo per se, es decir, a un fenómeno que tiene un determinado grado de regularidad en la secuencia temporal y duración de las fases de auge y contracción. Es en el contexto de crisis que Keynes se refiere a fluctuaciones violentas y bruscas, del tipo que Prebisch tenía en mente para describir el ciclo como fases de auge y colapso (Keynes, 1936: 314; Prebisch, 1947: 71; Pérez Caldentey y Vernengo, 2011 y 2012). No obstante, desde un principio Keynes enfatizó la importancia de la dinámica y esto tenía obviamente un impacto en su análisis del ciclo, aunque no desarrolló con profundidad este aspecto. ${ }^{13}$

Pero lo más interesante, y que atañe directamente a la visión que tenía Prebisch del capitalismo, es la descripción que hace Keynes de las economías de mercado como sistemas en un estado de perpetuo movimiento. Así, por ejemplo, enfatizó que de manera más general el cambio en las expectativas puede causar fluctuaciones similares a las de un ciclo económico: «[...] un simple cambio en las expectativas es capaz de producir una oscilación de la misma clase y perfil que un movimiento cíclico». Más aún, las economías de mercado aparecían como sujetas a cambios continuos: «El estado de las expectativas está sujeto a un constante cambio, superponiéndose una nueva expectativa antes de que la anterior se haya desenvuelto por completo. De manera que la maquinaría económica está ocupada todo el tiempo por actividades que se traslapan y cuya existencia se debe a estados pasados de expectativas» (ibid.: 50). Así, el volumen y nivel de empleo dependen, en todo momento, de los estados de expectativas que hayan existido en un determinado periodo de tiempo.

Pese a su importancia para entender tanto el mensaje como el método de la Teoría general, Prebisch no incluyó este capítulo en su Introducción. Tampo-

${ }^{113 /}$ El ejemplo más ilustrativo es el tratamiento de las expectativas, que es el tema central del capítulo quinto de la Teoría general, en el que distingue dos tipos de expectativas, las de corto plazo y las que atañen al largo plazo. En sus discusiones con Harrod, a raíz de la publicación del artículo sobre la dinámica de éste último en el Economic Journal, del cual era editor, Keynes deja claro que es contrario a formalizaciones mecánicas de la dinámica y muestra su rechazo de la noción del acelerador (cw, vol. xiv: 321-50). 
co menciona las expectativas de corto plazo. Finalmente, hace breve referencia al capítulo 12 de Keynes que trata sobre las expectativas de largo plazo, pero en el contexto de la relación entre eficiencia marginal y los mercados financieros. No cabe duda que la Teoría general tuvo un impacto significativo y duradero en el pensamiento de Prebisch, pero éste fue a la vez negativo y positivo. Por una parte, tal y como explicaremos más adelante, Prebisch nunca aceptó algunas partes esenciales del pensamiento de Keynes. Cuestionó fuertemente la explicación del desempleo basado en el carácter monetario de la tasa de interés y la lógica y la utilidad del multiplicador.

Por otra parte, rescató el diagnóstico de Keynes con respecto a las «fallas salientes de las economías de mercado: el no haber podido lograr la plena ocupación, y la distribución arbitraria de las riquezas e ingresos». A éstas, Prebisch añadiría una tercera falla, a saber, la inestabilidad. Lo que se condice con la importancia que Prebisch otorgó al ciclo como característica inherente del funcionamiento de las economías de mercado (Prebisch, 1991, vol. III: 500; vol. IV: 301-303; 344; 1947: 137). Si bien Keynes enfatizó las severas fluctuaciones del producto y empleo, fue cuidadoso en recalcar que el sistema no era «violentamente inestable» (1936:245), y que las fluctuaciones se daban alrededor de un nivel sub-óptimo. En este contexto, cabe recordar el famoso pasaje del capítulo 18 de la Teoría general, según el cual la economía:

[...] oscila, evitando fluctuaciones extremadas en ambas direcciones del empleo y de los precios, alrededor de una posición intermediaria, apreciablemente abajo del pleno empleo y apreciablemente por arriba del nivel que pondría en peligro la reproducción (ibid.: 254)

Además, según Keynes, la inestabilidad del sistema no depende de oleadas de psicología irracional y el estado de las expectativas es, por lo general, firme (p. 162), siendo que las decisiones se basan en convenciones, lo que de hecho les da cierta estabilidad ( $\mathrm{p}$. 152) por el mero hecho que implica que la situación prevaleciente continúa de manera indefinida, esto pese a su «preca- 
riedad». Prebisch, capaz porque provenía de un país periférico, aunque por cierto no negaría que las fluctuaciones se daban alrededor de niveles sub-óptimos, parecía creer en una inestabilidad más acentuada de las fluctuaciones del sistema capitalista.

\section{LA CRISIS DE LA TEORÍA ECONÓMICA Y LOS LÍMites de la TeOría general}

En 1948, con posterioridad a la publicación de la Introducción a Keynes, Prebisch argumentó que la teoría económica atravesaba una grave crisis. El atribuía esta crisis al hecho de que, en resumidas cuentas, la teoría económica no tenía ningún correlato con la realidad («la economía [la teoría económica prevaleciente] es insuficiente para abarcar, describir, y explicar los problemas de la realidad, y por lo tanto, para darnos los medios para actuar sobre ella con eficacia» (ibid.: 496). Prebisch incluyó en esta categoría al grupo de pensadores que Keynes, en su Teoría general, denominó la Escuela de Economía Clásica, pero que corresponde a la teoría dominante del momento, o sea, la teoría neoclásica. Además, Prebisch también puso al propio Keynes en esta misma categoría, en parte, como vimos, porque Keynes parecía más preocupado con los niveles de empleo que con la dinámica cíclica.

Prebisch pensó a lo largo de su vida que el proceso de crecimiento de las economías capitalistas, lejos de ser lineal, era eminentemente cíclico. Como él decía (Prebisch, 1991, vol. III 499):

[...] el ciclo es la típica forma de crecimiento que el capitalismo ha tenido históricamente y sigue teniendo. La actividad económica [...] se expande y contrae continuamente en una sucesión interrumpida de las fases de crecimiento en los ingresos, en la ocupación y en la producción, seguida de fases de decrecimiento con la consiguiente declinación de la producción y la ocupación. No hay punto de reposo: se asciende para descender 
y se desciende para ascender nuevamente [...] En ese movimiento no hay punto de equilibrio [...] es una sucesión continua de desequilibrios.

Esta visión del capitalismo no era exclusiva de Prebisch. De hecho tiene alguna similitud con un teórico del ciclo contemporáneo, Joseph Alois Schumpeter (1883-1950). ${ }^{14}$

De manera general, Prebisch desestimaba las explicaciones vigentes del ciclo basadas en el mal funcionamiento del sistema monetario, en causas exógenas y lo que hoy se denomina «choques» o en argumentos fundamentados en imperfecciones de mercado. Para Prebisch, en cambio, el ciclo es un resultado lógico y natural del funcionamiento de las fuerzas del mercado. Y más que un desperfecto resultante de fallas institucionales o de otra naturaleza, es un fenómeno endógeno y de hecho un fiel reflejo de su funcionamiento natural y lógico (i.e., «un fenómeno inherente al propio proceso capitalista; que el ciclo es el resultado lógico, espontáneo, de la forma en que actúan los empresarios, y que éstos no llevan, en su forma de actuar, al equilibrio, sino precisamente, a la sucesión de desequilibrios», vol. III: 502). ${ }^{15}$

Son justamente los problemas con la concepción de la teoría económica que lo llevan a Prebisch, con posterioridad a su Introducción a Keynes (1947), a realizar fuertes críticas explícitas a la Teoría general, no sólo tildándola de

/14/ Véase Schumpeter (1934: 214-215, 1939). Sin embargo, Schumpeter creía que la economía después de cada choque al flujo circular, provocado por las innovaciones, volvería al flujo circular donde la teoría walrasiana del equilibrio general explicaría el funcionamiento de la economía. Este tipo de análisis es muy diferente al propuesto por Prebisch, aunque ambos tengan una preocupación con la dinámica del capitalismo. Sin embargo, en los elementos marxistas del análisis schumpteriano, o sea, en su visión del capitalismo como un proceso de constante destrucción creativa, hay una confluencia con el pensamiento de Prebisch. En particular, la visión de Prebisch sobre el capitalismo periférico es lo que hace su análisis tan significativo y original, como discutiremos en la próxima sección.

${ }^{15 /}$ Los modelos de ciclos no lineales de acelerador-multiplicador de Kaldor (1940) y Goodwin (1951) se aproximan a la concepción prebischiana, aunque son más mecánicos y no captan la complejidad de la relación centro y periferia. 
inapta como instrumento para explicar la realidad, sino como un retroceso en el pensamiento de Keynes. Así, en junio de 1948, Prebisch escribe acerca de Keynes (Prebisch, 1991, vol. III: 506): «que sus teorías fundamentales son inaptas para explicar la realidad cíclica, es decir, la forma en que se desenvuelve el proceso económico en el mundo capitalista».

Pese a su crítica, Prebisch resaltaba la importancia de Keynes en el plano de la política económica de su duradero legado y llegó a vaticinar proféticamente el cíclico de las ideas keynesianas. Para él: «si sobreviniese una nueva depresión económica, Keynes llegaría a adquirir [...] la misma notoriedad que tuvo en la gran depresión mundial» (Prebisch, 1991, vol. III: 506). Pero para Prebisch las recomendaciones de Keynes en materia de política económica eran independientes de su teoría. En sus palabras: «Keynes ha dejado soluciones prácticas que son independientes de su teoría [...] puede admitirse o rechazarse con completa independencia de su teoría». Así, afirmaba que «no hay contradicción entre mi posición teórica para juzgar a Keynes y mi respeto por algunas de sus proposiciones prácticas» (ibid.: 506).

La crítica central de Prebisch al Keynes de la Teoría general fue su incapacidad para emanciparse de la lógica del análisis neoclásico para explicar la persistencia del desempleo, la determinación del nivel de producto y el ciclo. Keynes y su grupo de colaboradores más cercano, el llamado Circus de Cambridge, consideraron el pensamiento de la Teoría general novedoso y que marcaba una clara ruptura con sus planteamientos anteriores, en particular con el Tratado sobre el dinero (1930), aunque algunos de los elementos más novedosos - como el multiplicador, la preferencia por la liquidez o el concepto de tipos de interés propios - tienen claramente antecedentes en obras anteriores al magnum opus de Keynes.

Keynes resaltó, a lo largo de su obra y de distintas maneras, la ruptura de la Teoría general con la teoría económica precedente. En el prefacio a la Teoría general, escrito en diciembre de 1935, Keynes afirmó que la composición del libro había sido una larga lucha de escape de los modos habituales de pensamiento y expresión, enfatizando que la dificultad no reside en nuevas ideas 
sino en escapar de las viejas ideas que están ramificadas en cada rincón de nuestro cerebro, «de quienes se han educado en ella como la mayoría de nosotros». Esto es justamente lo que Prebisch negaba. Para Prebisch, la Teoría general no marcaba un hito de ruptura con los modos habituales de pensamiento. Se trataba más bien de una obra que, pese a sus intenciones y propósitos, mantenía la continuidad teórica y conceptual con la teoría neoclásica. De manera más precisa, Prebisch hizo dos críticas fundamentales a la Teoría general. La primera es que Keynes descansaba en rigideces y en particular en la tasa de interés para explicar el desempleo. La segunda es que el análisis del multiplicador era un artificio para obviar la inclusión del factor tiempo en sus análisis.

Para Prebisch, la explicación del desempleo involuntario de Keynes se sustentaba en un argumento imperfeccionista, a saber, la rigidez de la tasa de interés. En este sentido, Prebisch no veía diferencia alguna entre este argumento de Keynes y el argumento neoclásico también sustentado en una rigidez, la rigidez de los salarios nominales. Al fin y al cabo las posiciones de subempleo se explicaban por la inflexibilidad de un precio nominal. Tal y como argumentó Prebisch (1991, vol. III* 501):

El mismo Lord Keynes, que hizo un gran esfuerzo para emanciparse de las enseñanzas [neo]clásicas, cae también en un tipo de explicación eminentemente [neo]clásica. ¿Cuál es la explicación de Keynes, con relación al caso [la desocupación] que he citado precedentemente? Sencillamente, que cuando el tipo de interés no baja en la medida en que ha caído el rendimiento marginal del capital, o eficiencia marginal del capital, queda una parte del ahorro de la colectividad sin emplear y se produce la desocupación. Para los economistas clásicos anteriores a Keynes, la rigidez de los salarios, [...] provoca la desocupación. Por otro lado, Keynes, al pretender salirse de los [neo]clásicos cae en un tipo de explicación eminentemente clásica de la desocupación. A mi juicio, ambas están equivocadas porque en realidad no se presentan los fenómenos en esa forma. 
Y más adelante Prebisch explica de la misma manera (Prebisch, 1993, vol. IV: 276): «¿en qué estriba la explicación de Keynes? En que el tipo de interés se resiste a caer cuando baja el rendimiento del capital. Son explicaciones que están dentro del juego lógico de la escuela [neo]clásica». Prebisch sostuvo que la preferencia por la liquidez explicaba, según Keynes, la rigidez a la baja de la tasa de interés. No obstante, Prebisch parece haber tenido en mente un fenómeno más parecido a la «trampa de la liquidez» que la preferencia por la liquidez per se. Esto se desprende de su propia explicación (ibid.: 275): «¿por qué razón a juicio de Keynes deja de bajar la tasa de interés? Por el fenómeno de preferencia de liquidez, que se produce cuando la tasa toca un nivel demasiado bajo y los inversores temen que al venirse tan abajo puede en cualquier momento volver a subir con los efectos que ello tendría en su colocación». Por consiguiente, para Prebisch, mientras más baja es la tasa de interés, mayor su resistencia a disminuir y mayor la posibilidad de que los agentes económicos mantengan su nivel de liquidez evitando un aumento de la demanda agregada. ${ }^{16}$

De hecho, para Keynes la preferencia por la liquidez hacía referencia al volumen de recursos (valorados en términos nominales o unidades de salario) que un agente mantiene bajo la forma de dinero en distintas circunstancias (1936: 166). Mientras la «trampa de la liquidez» se refiere a una situación en la cual la preferencia por la liquidez es absoluta en el sentido de que la tasa de interés ha caído a un nivel tan bajo que todos prefieren mantener el dinero, el activo de liquidez absoluta, a conservarlo en un bono con un rendimiento tan bajo. Keynes fue enfático al declarar que si bien esta podía ser una posibilidad futura, no conocía ningún caso práctico de su existencia (1936: 207).

En la Teoría general, Keynes asignó un papel central a la tasa de interés. Para Keynes, según la teoría neoclásica, la flexibilidad de los salarios es una condición necesaria pero no suficiente para lograr posiciones de pleno empleo, se requería además el ajuste de la tasa de interés (Pigou, 1944: 16). Keynes

/16/ Para una visión moderna de la trampa de la liquidez, véase Krugman (1998). 
criticó las bases teóricas sobre las cuales se sustentaba la flexibilidad de los salarios y argumentó que los efectos de la flexibilidad de los salarios en el nivel de empleo y producto podían ser contrarios a los postulados por la teoría clásica. No obstante, la objeción principal de Keynes radicaba en la imposibilidad de que al tipo de interés se ajustara de tal forma a lograr posiciones de equilibrio con pleno empleo (i.e., con desempleo voluntario). De hecho, en su capítulo sobre la flexibilidad de los salarios, Keynes (1936:266) argumentó que: «es sobre los efectos de disminuciones salariales y en los niveles de precios sobre la demanda de dinero que quienes creen el carácter automático del sistema económico deben centrar el peso de su argumento». El argumento principal de Keynes, que sustentaba la falta de ajuste en el tipo de interés, es que éste, lejos de ser un fenómeno real, como postulaba la teoría clásica, era más bien un fenómeno monetario. ${ }^{17}$

Keynes argumentó que al ser un fenómeno monetario, el tipo de interés establecía una referencia hacia cuál debían ajustarse las eficiencias marginales de los bienes de capital para que éstos puedan volver a producirse. Prebisch interpretó a Keynes de manera contraria asumiendo que el tipo de interés debía ajustarse a las eficiencias marginales de los bienes de capital. Sólo esta interpretación es compatible con la afirmación anterior de Prebisch (1991, vol. III* 501), según la cual Keynes sostenía: «[...] que cuando el tipo de interés no baja en la medida en que ha caído el rendimiento marginal del capital, o eficiencia marginal del capital, queda una parte del ahorro de la colectividad sin emplear y se produce la desocupación».

${ }^{/ 17 /}$ Es importante resaltar que Keynes, aunque explícitamente criticaba la noción neoclásica (wickselliana) de la tasa natural de interés, como vimos, mantenía la idea de la eficiencia marginal de capital que presupone que hay una tasa de interés suficientemente baja para ecualizar la inversión con la tasa de ahorro de pleno empleo (Camara Neto y Vernengo, 2012). O sea, hay una tasa real que permite el pleno empleo. En realidad, el argumento según el cual el pleno empleo no ocurre está basado en el capítulo 17 de la Teoría general. Keynes argumenta que las características del dinero y la tasa de interés propia del dinero son los que imponen el límite al sistema económico antes que éste alcance el pleno empleo. 
En el capítulo 17 de la Teoría general, Keynes explica que todos los bienes tienen un rendimiento esperado que se puede expresar en términos de sus propias unidades. La igualdad entre distintos tipos de interés propios, expresados en dinero para un determinado grupo de bienes o activos, implica que los agentes son indiferentes entre éstos. No hay una ventaja relativa entre la tenencia de un activo (o bien) y de otro. Ésta es una situación de equilibrio de acervos. El razonamiento de Keynes puede entonces explicarse suponiendo que existen dos activos, uno monetario (el dinero) y otro activo. Por tanto, la tasa propia de interés del dinero se fija mediante la decisión de una autoridad monetaria mientras que la tasa propia de interés del activo varía inversamente con la cantidad de unidades producidas debido a la existencia de rendimientos marginales decrecientes. En una economía en expansión tiende a disminuir el rendimiento de los activos utilizados más intensamente. La suposición neoclásica para Keynes era que todos los activos serían producidos hasta el punto que comportaría la plena utilización de los recursos productivos, si no fuera por la existencia del dinero, un activo con características especiales.

El punto central para Keynes es que la tasa de interés propia del dinero no tiene una tendencia a la baja cuando la economía crece, porque tendría una elasticidad cero de producción y de sustitución. $\mathrm{O}$ sea, se puede producir más dinero sin incrementar los costos de producción, con lo que no hay rendimientos decrecientes, y los agentes no buscan otros activos, ya que la producción de dinero es de bajo costo. Si la tasa de interés propia que permite la plena producción de un activo cae por debajo de la tasa propia del dinero, entonces el desempleo sería posible. Así, Keynes (1936: 235) concluyó: «en ausencia del dinero debemos por supuesto suponer de cualquier otro bien con las supuestas características del dinero, las tasas de interés llegarían al equilibrio sólo cuando hay pleno empleo».

Prebisch se mostró crítico de esta interpretación. Para Prebisch, el fenómeno que describe Keynes (Prebisch, 1947: 110): «puede ocurrir independientemente del dinero, siempre que exista algún bien cuya tasa de interés, en sus propios términos, encuentre dificultades para bajar más allá de ciertos lí- 
mites cuando aumenta su cantidad, aun en una economía sin dinero» ${ }^{18}$ Quizá este tipo de interpretación lo llevó a afirmar «que hay épocas en la historia en que el deseo de tener tierra ha desempeñado un papel similar al que hoy tiene el dinero en mantener elevado el tipo de interés» (ibid.: 112). Desde nuestro punto de vista, lo importante de la crítica de Prebisch a Keynes es la percepción correcta de que la ruptura con la teoría dominante debe necesariamente romper con argumentos imperfeccioncitas. No es evidente que Prebisch tuviera claro, como Keynes lo tenía, los problemas con la tasa natural de interés, pero supo criticar correctamente la noción de que una rigidez a la baja de la tasa de interés pudiera ser vista como la causa del desempleo.

\section{El MULTIPLICADOR LÓGICO Y LA VELOCIDAD DE CIRCULACIÓN DEL DINERO}

La segunda crítica de Prebisch se centró en lo que Keynes llamó la novedad inicial de la Teoría general (cw, xiv ₹212). Esto es la igualación de la inversión y el ahorro por variaciones en el nivel de producto y no en el tipo de interés. De manera más precisa, Keynes argumentó que la inversión es independiente del ahorro y determina a través de la propensión marginal a consumir (su ley psicológica esencial, 1936: 113-131, cw, xxix :214) y el multiplicador el nivel de ingreso al cual se ajusta el ahorro.

En un principio, las críticas de Prebisch se centraron en afirmar que el efecto multiplicador del que hablaba Keynes sólo funcionaba en una economía cerrada, con referencias marginales a la propensión a importar, sin referencia

${ }^{/ 18 /}$ En este pasaje, Prebisch parece próximo de la crítica de Sraffa, el autor que había introducido Keynes a la noción de las tasas propias de los activos en su debate con Hayek, según el cual la tasa propia del dinero no es diferente de la de otros activos. Sraffa, sin embargo, argumentaba que el problema de Keynes residía en la aceptación del concepto de productividad marginal de los activos. Para una discusión de la crítica de Sraffa al capitulo 17 de la Teoría general, véase Kurz (2011). 
alguna a la velocidad de circulación del dinero y limitado principalmente en sus efectos por la propensión a ahorrar, «lo que constriñe la expansión de la actividad económica y conspira contra la plena ocupación» (Prebisch, 1991, vol. III: 359). En su Teoría general, Keynes se interesó especialmente en la economía empresarial y el proceso de toma de decisiones en un entorno incierto. En este sentido, el análisis del multiplicador adopta una forma superficial y hasta quizá incompleta (Kahn, 1984: 134; Chick, 1997: 162-184). No obstante, conocía muy bien los efectos de la propensión a importar en el multiplicador, como queda demostrado en el razonamiento detrás de su estimación del multiplicador de Gran Bretaña y la comparación con Estados Unidos (Keynes, 1933). ${ }^{19}$

Esto es consecuencia de que la propensión a importar formaba parte del marco y de la lógica con que fue concebido el multiplicador (Kahn, 1972). Las importaciones fueron consideradas - junto con los ahorros y la porción que no se transfiere de la renta de los desempleados - un derrame y los derrames garantizaban que el multiplicador podía expresarse como una serie geométrica infinita, pero convergente. En consecuencia, la obra de Keynes Los medios para la prosperidad, publicada tres años antes que la Teoría general, donde se habla de una economía abierta, incorpora la propensión a importar como parte del análisis del multiplicador. Además, muchos autores del momento, como Giblin (1930), Warming (1932), Kalecki (1933) y Harrod (1933), abordaron el tema del multiplicador del comercio exterior. En 1941, unos tres años antes de que Prebisch analizara en profundidad el tema del coeficiente de expansión de las exportaciones, el multiplicador del comercio exterior ya era un concepto muy afianzado en la literatura (Haberler, 1945).

/19/ En los trabajos de Keynes se observa una gran preocupación por el sector externo. De hecho, en su Teoría general afirmó que la falta de interés por la posición externa de un país era consecuencia del laissez-faire y expresó: «[...] el peso de mi crítica contra lo inadecuado de los fundamentos teóricos de la doctrina del laissez-faire, en la que fui educado [...] contra la idea de que la tasa de interés y el volumen de inversiones se ajustan automáticamente al nivel óptimo, de manera de que preocuparse por la balanza comercial sea perder el tiempo» (Keynes, 1936: 319). 
Más adelante, Prebisch puso en tela de juicio la forma mediante la cual Keynes determinaba el producto y el empleo, afirmando que la inversión no determinaba el ahorro tal y como lo proponía Keynes: «Estoy convencido, que el dejar de consumir es esencial para capitalizar inversiones. En esto estoy de acuerdo con las razones que expone la escuela [neo]clásica y discrepo de Keynes» (Prebisch, 1993, vol. Iv: 299). Esto lo llevó obviamente a criticar la forma mediante la cual Keynes determinaba el volumen de producto y empleo, es decir, el multiplicador. La objeción fundamental de Prebisch es que Keynes, al igual que los neoclásicos, prescindían del factor tiempo en sus teorías, que era un factor fundamental para explicar el funcionamiento de las economías de mercado. Ambos, de hecho, introducían artificios para este fin. En el caso de los clásicos, el tipo de interés era el artificio por excelencia; en Keynes, el multiplicador. Prebisch expone la razón de su discrepancia con Keynes en los siguientes términos (ibid.: 277).

[...] en el fondo el pensamiento keynesiano significa decir: para hacer inversiones hoy, usaremos como ahorro el que se haga en el futuro [...] Este razonamiento puede hacerse si se confunde el futuro con el presente eliminando completamente el factor tiempo en el proceso. Tal es una de las grandes inconsistencias que invalidan la teoría keynesiana. Ahí entramos en la más profunda discrepancia entre Keynes y la escuela clásica. Usando un símil, para los clásicos, a fin de producir un huevo, dejar hoy de consumir un huevo. Todo esto no es aceptado por Keynes. ¿Porqué tenemos que ahorrar hoy un huevo para producir esa gallina y no producimos esa gallina con el huevo que ella misma va a poner en el futuro? Eso es en el fondo, a mi juicio, la teoría keynesiana del multiplicador.

En otras palabras, Prebisch es crítico de lo que Keynes (1936: 122) se refería como la teoría lógica del multiplicador, que está relacionada con la lógica del principio de la demanda efectiva, i.e., la determinación del ahorro por la inversión de un punto de vista lógico, cuando la inversión es tomada como 
genuinamente autónoma de los ingresos y no por el proceso histórico en el cual esto ocurre con rezagos y procesos simultáneos de gastos y generación de ingresos. De hecho, Prebisch criticó el multiplicador y el proceso asociado de ahorro-inversión por considerarlos una representación intemporal de las economías capitalistas (Prebisch, 1993, vol. IV: 277), de poca relevancia para los países en desarrollo. ${ }^{20}$ En principio, se podría decir que la preocupación con los procesos históricos de desarrollo en la periferia, y la misma dificultad a la que aludía Keynes de alejarse de la ideas convencionales, en parte explica estas críticas de Prebisch al multiplicador keynesiano.

Pese a estas críticas, Prebisch desarrolló de hecho un concepto muy similar, sino idéntico, al multiplicador del comercio exterior alrededor de 1935 y denominado por Prebisch el «coeficiente de expansión» (Prebisch, 1991, vol. III: 249-298, 301-310, 335-342 y 349-370). ${ }^{21}$ Según Prebisch, el coeficiente de expansión medía la intensidad con que un aumento de la renta, causado por un incremento de las exportaciones o los flujos financieros, produce una mayor expansión de la actividad económica interna. Su análisis del multiplicador del comercio exterior era estático, una explicación del cambio de una posición de equilibrio a otra.

A partir de una posición de total equilibrio, explica el funcionamiento de un único aumento de las exportaciones de la siguiente manera (Prebisch, 1991, vol. III: 250):

Si por ejemplo el volumen de las exportaciones argentinas aumentase - ya sea por el crecimiento de la cantidad exportada o por el aumento de los precios- el sector agropecuario recibirá ingresos correlativamente mayores que le permitirán aumentar su demanda de los artículos y

\footnotetext{
120/ En 1949, Prebisch aún sostenía que los ahorros determinaban la inversión y que uno de los principales problemas de las economías en desarrollo, como la de Argentina, era la falta de ahorros. Véase Prebisch (1991, vol. III, 361 y 367). Sobre el multiplicador lógico de Keynes, véase Bortis (2008).

121/ Véase también Fernández-López (1996).
} 
servicios producidos en los otros sectores y asimismo de los artículos importados. Habrá una mayor demanda de artículos industriales; más actividad en el comercio y en los transportes; mayor utilización de servicios profesionales [...] y mayores importaciones. A su vez, esos sectores que habrán recibido mayores ingresos aumentarán su demanda de artículos y servicios producidos dentro del mismo sector y en otros sectores, y así sucesivamente se irá ampliando la influencia o el efecto del crecimiento inicial de ingresos del sector agropecuario, producido por el aumento de sus exportaciones.

Al final, el sistema vuelve al punto de equilibrio cuando el incremento de la renta nacional, causado por el crecimiento de las exportaciones, llega al resto del mundo mediante un mayor volumen de importaciones y otros pagos. En los ejemplos que presenta Prebisch, la renta se gasta íntegramente en el país o en el exterior con las importaciones. Como resultado, la propensión marginal a ahorrar es, en última instancia, igual a cero y el efecto de un cambio en las exportaciones en el gasto se reduce al inverso de la propensión marginal a importar o al multiplicador del comercio exterior. Por lo tanto, el aumento de la renta está determinado por el incremento de las exportaciones multiplicado por el multiplicador del comercio exterior. Formalmente, como en el multiplicador lógico de Keynes, el nivel de la renta es determinado por un cambio autónomo en las exportaciones. Prebisch contrastó el coeficiente de expansión con el multiplicador keynesiano argumentando no sólo que Keynes ignoraba la existencia del sector externo sino destacando otro factor limitante de la potencia del «coeficiente de expansión»: la velocidad de circulación del dinero, es decir, la cantidad de veces que el dinero cambia de manos. ${ }^{22}$

Antes de que se publicara la Teoría general, Clark (1935: 16) había distinguido dos enfoques: uno mediante ciclos sucesivos de ingreso y gasto por los re-

122/ John Maurice Clark también destacó la importancia de la circulación monetaria para el funcionamiento del proceso multiplicador (Fiorito y Vernengo, 2009). 
ceptores finales de la renta y otro mediante el volumen de dinero y su velocidad de circulación. Clark recordó que el primero había sido desarrollado por Kahn y Keynes, y el segundo, hasta donde él sabía, no había llegado a publicarse.

Sobre la base de esta distinción de Clark, algunos autores declararon que la lógica del multiplicador incluía implícitamente suposiciones respecto del comportamiento de la velocidad de circulación del dinero y que el análisis estaba incompleto sin su explícita incorporación. Haberler (1945) sostenía que para determinar los efectos secundarios del nuevo gasto público era necesario contar con información sobre la propensión marginal a consumir y la velocidad de circulación del dinero. Machlup (1939), por su parte, se refirió a la gran importancia del elemento tiempo para la teoría del multiplicador e introdujo el análisis por etapas para entender los efectos primarios y secundarios de las inversiones en obras públicas, donde los periodos se consideran recíprocos de la velocidad de circulación del dinero. Prebisch (1991, vol. III: 359) parece tener una idea similar, ya que afirma que tras el incremento de la renta, el empleo primario crecerá, pero eso no producirá una expansión del empleo secundario a menos que haya otra ronda de inversiones o que aumente la velocidad de circulación del dinero.

\title{
Bretton Woods, COMO SI HUBIERA CENTRO Y PERIFERIA
}

\begin{abstract}
A medida que Prebisch fue desarrollando y refinando su crítica a la teoría convencional y a Keynes, con independencia de su tino, se dio cuenta, por un lado, de la necesidad de contar con una teoría que incluyera como pivote central en sus distintas dimensiones y aspectos justamente lo que la teoría económica no había sido capaz de incorporar, a saber, el factor tiempo, y una perspectiva que llevaría a lo que se ha denominado el método histórico/institucional, con el que ha quedado identificado el pensamiento estructuralista latinoamericano. Pero hay que entender que la preocupación con la temporalidad y la institucio-
\end{abstract}


nalidad específica de los procesos que ocurren en tiempo histórico eran esenciales para los presupuestos teóricos de Prebisch. En sus palabras:

Fue precisamente esta preocupación la que me fue dominando en mis anteriores actividades prácticas, e influyó después para hacerme buscar sistemáticamente la acción del tiempo no sólo en los fenómenos de circulación de ingresos, sino en otros fenómenos de la economía [...] y así [...] he ido llegando a esta conclusión fundamental de la cual se deriva toda la teoría que voy a exponer (Prebisch, 1993, vol. Iv: 416).

Esto fue los que lo llevó a desarrollar su Teoría de la Dinámica (véase Vernengo y Pérez Caldentey, 2012b).

Por otra parte, tomó cada vez más conciencia de la interrelación e interconectividad entre los distintos países y las distintas regiones, y en particular la relación asimétrica entre centro y periferia y sus respectivos roles en la división internacional del trabajo. ${ }^{23}$ En otras palabras, una concepción sofisticada del proceso de desarrollo histórico del capitalismo ya queda evidente en los trabajos teóricos de Prebisch que preceden el «Manifiesto desarrollista» de $1949 .{ }^{24}$ Esto le permitió entender tanto el ciclo como la dinámica desde una perspectiva global y, más precisamente, bajo la óptica de la relación cen-

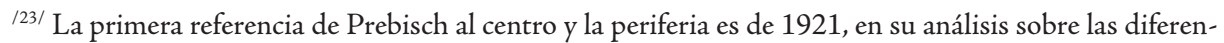
cias de la colonización en el Río de la Plata y en Estados Unidos (1991, vol. i: 149). Esto no quiere decir que la concepción del desarrollo simbiótico entre el centro y la periferia, conectados por los procesos de crecimiento cíclico, ya lo tuviera claro Prebisch en 1921. Pero es evidente que desde muy temprano tenía una preocupación con la periferia como un ámbito económico específico.

124/ Crotty (1990) sugiere que Keynes también tenía una visión más sofisticada sobre la evolución histórica e institucional del capitalismo avanzado de lo que generalmente se supone. Pero, aunque tuviera razón Crotty, es todavía verdad que no hay en Keynes, o ningún autor teórico relevante de la época, una comprensión sobre los problemas de los países periféricos. La idea de que sólo se puede entender el ciclo en la periferia como complemento del ciclo en el centro es, en ese sentido, una contribución de Prebisch al desarrollo de la economía como disciplina. 
tro-periferia. En lo que concierne a su relación con las ideas de Keynes, esta sensibilidad histórica llevó a Prebisch a preocuparse por los efectos que en la periferia podían tener los planes económicos y monetarios internacionales elaborados por y para los países del centro. Estos incluían tanto los planes de Keynes como los de Harry Dexter White y de John H. Williams. ${ }^{25}$

Prebisch fue crítico de los planes británico y estadounidense. Comparó al plan de Keynes (La Unión de Compensaciones) con un «Fondo Monetario Internacional Cerrado» (Prebisch, 1993, vol. IVः 97). Esto porque la liquidez generada por los países de la Unión de Compensaciones se gastaba en las transacciones comerciales entre éstos. Ello no permitía la creación de un sistema comercial equilibrado, equitativo y beneficioso para la periferia. ${ }^{26}$ Más aún, Prebisch estimaba que el plan de Keynes adolecía de las falencias que caracterizaban al patrón oro y, más específicamente, de un automatismo «que tanto daño ha hecho al sistema universal de Patrón Oro» (ibid.: 99). Este automatismo radicaba en que cada país podía hacer uso libre de los créditos acordados y Prebisch pensaba que esto conllevaba un peligro inflacionario. Esto obviamente tendría un efecto negativo sobre los países de la periferia. ${ }^{27}$ Por lo demás, Prebisch pensaba que los créditos que provee un sistema internacional debían dirigirse a satisfacer las necesidades imperantes de los países, incluyendo el caso de los países de la periferia.

Las críticas más duras, no obstante, se dirigieron al plan estadunidense de White. Éste era aún menos favorable para los países de la periferia, y no sólo

125/ Véanse Prebisch (1993, vol. iv: 94-112) y Asso y Fiorito (2004).

126/ En sus palabras: «[...] no obstante sus grandes méritos, [los proyectos de compensación] no resuelven el problema de fondo que depende esencialmente de la restauración del comercio internacional. Si Estados Unidos no compran del resto del mundo tanto como el resto del mundo compra de Estados Unidos [...] no habrá sistema monetario que resista a la larga» (Prebisch, 1943: 8-9).

127/ Prebisch señaló el peligro de «la afluencia excesiva de dinero nuevo proveniente del uso del crédito internacional sobre la economía de Estados Unidos» (ibid.: 100). A la luz de la experiencia reciente actual, sobre todo en los que concierne a la crisis financiera global (2007-2009), se podría matizar la afirmación de Presbich argumentando que el automatismo podría conllevar a una inflación de activos. 
no resolvía el problema de la inflación sino que además, pese a aliviar temporalmente los desequilibrios de la balanza de pagos, era en el fondo un plan de contracción. Esto podía impactar fuerte y negativamente en el crecimiento del producto y del empleo en la periferia. Como señaló Prebisch (ibid.: 103-104):

Supongamos, que en Estados Unidos se produzca una fuerte contracción económica y del crédito como ocurrió después de la crisis mundial o [...] después de la primera guerra. Supongamos que posteriormente la expansión sea relativamente lenta [...] ¿Qué ocurrirá si [...] los países de la periferia [...] ven un desequilibrio creciente de su balance de pagos? [...] Que los países afectados recurrirán a los créditos [...] pero esos créditos son limitados [...] para pagar sus créditos tendrán que recurrir a la contracción de sus importaciones [...] a la contracción de su economía interna.

Prebisch proponía una alternativa, un plan de plena ocupación en consonancia con la propuesta de Williams. Éste consistía en que el Estado, en los países del centro (y en particular Estados Unidos), compense la caída de la actividad privada con aumentos del gasto público, incluyendo obras públicas para sostener los niveles de producto y ocupación. Este plan incluía el manejo en la dirección del crédito para evitar desequilibrios insostenibles. Aunque Prebisch no lo recalcó, este plan era consistente con el planteamiento de Keynes en la Teoría general con respecto al ciclo sobre la necesidad de sostener las expansiones, un punto de vista al cual, según creemos, Prebisch llegó a esta conclusión un poco antes de dejar la gerencia del Banco Central de la República Argentina (BCRA) (Pérez Caldentey y Vernengo, 2011, 2012). Keynes argumentó en la Teoría general que uno de los desafíos principales con respecto al manejo del ciclo no era liquidar las expansiones sino tratar de sostenerlas en el tiempo. Tal y como lo afirmó (1936:322)ः «El remedio correcto para el ciclo económico no es abolir los auges y mantenernos en una situación permanente de semiestancamiento; sino más bien en abolir las recesiones manteniéndonos en un cuasi-auge continuo». 
Para Raúl Prebisch, los beneficios que llegara a tener el plan de pleno empleo serían «incalculables»:

El mundo podría tener un estímulo al crecimiento más o menos intenso que el que vimos en el siglo xIx, por la enorme potencia de crecimiento que ha tenido Estados Unidos, sobre todo si esa potencia va acompañada de innovaciones técnicas que sin duda ocurrirán [...] estamos muy lejos del agotamiento de las oportunidades de inversión, como algunos economistas puedan sostener, entre ellos el mismo Keynes (Prebisch, vol. IV: 108).

En particular, Prebisch destaca que la condición necesaria para que los planes funcionen es que el centro monetario funcione bien, porque, según él, «si el centro monetario funciona mal [...] serán absolutamente inútiles los planes monetarios» (ibid.: 96). Y para que funcionara bien el centro monetario, Prebisch indicaba que era «de capital importancia para la reconstrucción de la economía y del sistema monetario mundial [...] planes de plena ocupación en los principales países del mundo y especialmente en Estados Unidos». No obstante, no se mostraba optimista en cuanto a su posible adopción por las economías del centro. Esta visión perduraría en Prebisch incluso al orientar su análisis a partir de 1950 hacia los problemas de más largo plazo del desarrollo económico de América Latina, y la necesidad de estimular la demanda doméstica e impulsar la industrialización de la periferia.

\section{REFLEXIONES FINALES}

Keynes tuvo una profunda influencia en el pensamiento de Prebisch no sólo en términos del diagnóstico acerca de los principales fallos de las economías de mercado, sino también en la necesidad de llevar a cabo políticas anticíclicas. No obstante, el camino por el cual Prebisch desarrolló sus ideas no fue lineal, ni puede ser descrito como una aceptación acrítica de las ideas desarrolladas 
en los países centrales. De hecho, Prebisch fue un crítico bastante duro de la Teoría general. Prebisch sostuvo que la teoría de Keynes era inconsistente y no representaba en absoluto una ruptura con la lógica del análisis clásico. En particular, Prebisch correctamente nota que los argumentos centrales de la Teoría general son incapaces de escapar completamente de la visión imperfeccionista de los problemas del desempleo, en lo que tenía razón en última instancia, y a pesar del hecho de que Keynes también quería escapar de las explicaciones basadas en rigideces.

Las críticas de Prebisch se centraron en la teoría de interés y el multiplicador. De un lado, sugirió que el argumento de Keynes se asentaba incorrectamente en la rigidez a la baja de la tasa de interés. Prebisch no profundizó la crítica y no vio la relevancia del concepto de tasa de interés normal, que era monetaria convencional, discutido en la Teoría general, sin embargo su crítica al imperfeccionismo es, asimismo, relevante. Con relación al multiplicador, si bien por momentos en la discusión del multiplicador lógico Prebisch demuestra aceptar presupuestos pre-keynesianos con relación a la determinación de la inversión por el ahorro, queda claro en su tratamiento del coeficiente de exportación que defiende, como Keynes, la determinación del producto por el gasto autónomo, pero con una preocupación por el proceso institucional por el cual la expansión ocurre en el tiempo histórico.

La actitud de Prebisch en relación a Keynes puede explicarse por una diferencia en el objeto y método de análisis. Los intereses de Prebisch se centraban en la dinámica y los ciclos; temas periféricos al mensaje y análisis central de la Teoría general. Además, Prebisch tenía una concepción histórica del proceso de desarrollo, que diferenciaba el papel del centro y de la periferia, donde el ciclo económico en la periferia era resultado del ciclo en el centro dinámico. De ahí que no sólo Prebisch fuera crítico de la Teoría general, una obra que consideró alejada de la realidad de las economías capitalistas, particularmente en la periferia, sino más aún de los planes de reconstrucción de la posguerra. Sin embargo, a pesar de las críticas, no se debe dejar de notar que hay muchos puntos en común y confluencias entre los dos autores, y eso explica, en parte, 
la razón por la cual la escuela estructuralista, centrada en la Comisión Económica para América Latina y el Caribe (CEPAL), que Prebisch dirigió, sería vista como la versión latinoamericana de los keynesianos en el hemisferio norte.

\section{Referencias}

Amadeo, E. (1989), Keynes's Principle of Effective Demand, Aldershot, Edward Elgar.

Besomi, D. (2001), «Harrod's Dynamics and the Theory of Growth: The Story of a Mistaken Attribution», Cambridge Journal of Economics, 25(1), pp. 79-96.

Bortis, H. (2008), «The Multiplier Relation as the Pure Theory of Output and Employment in a Monetary Production Economy», en C. Gnos y L. P. Rochon (editores), The Keynesian Multiplier, Nueva York, Routledge, pp. 58-84.

Braun, C. R. (1986), «Debilidades en la edición del Fondo de Cultura de la “Teoría General" de Keynes», Investigaciones Económicas (segunda época), vol. $x$, núm. 2, pp. 411-417.

Camara Neto, A. y M. Vernengo (2012), «Keynes alter Sraffa and Kaldor: Effective Demand, Accumulation and Productivity Growth», en T. Cate (editora), Keynes's General Theory Seventy-Five Years Later, Cheltenham, Edward Elgar.

Cнiск, V. (1997), «The multiplier and finance», en G. H. Harcourt y P. A. Riach (editores), Maynard Keynes' General Theory, Nueva York, Routledge.

Clark, J. M. (1935), «Cumulative effects in aggregate spending as illustrated by public works», American Economic Review, vol. 25, núm. 1, Nashville, Tennessee, American Economic Association.

Сrotтr, J. (1990), «Keynes on the Stages of Development of the Capitalist Economy: The Institutionalist Foundation of Keynes's Methodology», Journal of Economic Issues, 24(3), pp. 761-80. 
Eichengreen, B. (1999), «The Keynesian revolution and the nominal revolution: Was there a paradigm shift in economic policy in the 1930s?», Berkeley, Universidad de California [en línea] 〈http»//www.econ.berkeley.edu/ eichengr/research/castronov.pdf>.

Fernández López, M. (2002), Recepción del pensamiento de Pareto en Argentina, Buenos Aires, Asociación Argentina de Economía Política (AAEP). (1996), «Ciclo económico periférico: los alumnos argentinos de Juglar», serie Seminarios, núm. 23, Buenos Aires, Instituto Torcuato Di Tella.

Fiorito, L. (2001), «John Maurice Clark's contribution to the genesis of the multiplier analysis», Quaderni, núm. 322, Siena, Universidad de Siena, mayo.

Fiorito, L.y M. Vernengo (2009), «The other J.M.: John Maurice Clark and the Keynesian Revolution», Journal of Economic Issues, vol. 43, núm. 4, Armonk, M. E. Sharpe.

Gesell, S. (1898), La anemia monetaria, Buenos Aires.

Goodwin, R. (1951), «The nonlinear accelerator and the persistence of business cycles», Econometrica, 19, pp. 1-17.

Haberler, G. (1945), Prosperidad y depresión, México, Fondo de Cultura Económica.

Hansen, A. H (1953), A Guide to Keynes, McGraw-Hill, New York.

Hayex, F. (1933), Monetary Theory and the Trade Cycle, Londres, Jonathan

Cape, publicado originalmente en alemán en 1929 como Geldtheorie und Konjunkturtheorie, Viena, Julius Springer Verlag.

Kahn, R+ (1984), The Making of Keynes' General Theory, Nueva York, Cambridge University Press.

(1972), «The relation of home investment to unemployment», Selected Essays on Employment and Growth, Cambridge, Cambridge University Press. Kaldor, N. (1940), «A Model of the Trade Cycle», Economic Journal, 50, pp. $78-92$.

Keynes, J* M. (1936 [2003]), Teoría general de la ocupación, el interés y el dinero, México, Fondo de Cultura Económica. 
Keynes, J. M. (1936) [1964]), The General Theory of Employment, Interest and Money, New York, Harcourt Brace Jovanovich Publishers (1933 [1997]), Los medios para la prosperidad, Barcelona, Editorial Folio. (1923 [1992]), Breve tratado sobre la reforma monetaria, México, Fondo de Cultura Económica.

(1971-1989), The Collected Writings of John Maynard Keynes, London, Macmillan, Cambridge University Press for the Royal Economic Society.

Krugman, P. (1998), «It's Baaack: Japan's Slump and the Return of the Liquidity Trap», Brookings Papers on Economic Activity, 2, pp. 137-205.

Kurz, H. (2011), «Keynes, Sraffa and the latter's “Secret Skepticism”», Nómadas, número especial.

Machlup, F. (1939), «Period analysis and the multiplier theory», Quarterly Journal of Economics, núm. 54, Oxford, Oxford University Press.

Mallorquín, C. (2007), «The unfamiliar Raúl Prebisch», Esteban Pérez Caldentey y Matías Vernengo (editores), Ideas, Policies and Economic Development in the Americas, Nueva York, Routdledge.

(2006), «Textos para el estudio del pensamiento de Raúl Prebisch», Cinta de Moebio, núm. 25, marzo, Santiago de Chile, Universidad de Chile.

Pérez Caldentey, E. (2003), «Chicago, Keynes and Fiscal Policy», Investigación Económica, LXII, pp. 15-45.

Pérez Caldentey, E. y M. Vernengo (2011), «Understanding the business cycle in Latin America: Prebisch's contributions», Estudios y perspectivas, núm. 127, México, Sede Subregional de la cepal en México, publicación de las Naciones Unidas.

(2012), «Portrait of the economist as a young man: Raúl Prebisch's evolving views on the business cycle and money, 1919-1949», CEPAL Review, 106, pp. 7-20.

Pigou, A. C. (1944), Lapses from Full Employment, London, MacMillan. Pollock, D., D. Kerner y J. Love (2002), «Aquellos viejos tiempos: la forma- 
ción teórica y práctica de Raúl Prebisch en la Argentina. Una entrevista realizada por David Pollock», Desarrollo económico, vol. 41, núm. 164, Buenos Aires, Instituto de Desarrollo Económico y Social.

Prebisch, R. (1993), Obras 1919-1949, vol. 4, Buenos Aires, Fundación Raúl Prebisch.

(1991), Obras 1919-1948, vols. 1 al 3, Buenos Aires, Fundación Raúl Prebisch.

(1986), «Cinco etapas en mi pensamiento sobre el desarrollo», Gerald Meier y Dudley Seers (editores), Pioneros del desarrollo, Madrid, Tecnos. (1950), Estudio económico de América Latina, 1949, Nueva York, Naciones Unidas.

(1949), El desarrollo económico de la América Latina y algunos de sus principales problemas, Santiago de Chile, Comisión Económica para América Latina y el Caribe.

(1947), Introducción a Keynes, Buenos Aires, Fondo de Cultura Económica. (1944) «Observaciones sobre los planes monetarios internacionales», El Trimestre Económico, vol. xi, núm. 2, julio-septiembre 1944.

(1943), La moneda y el ritmo de la actividad económica, inédito.

Schumpeter, J. A. (1939), Business Cycles, Filadelfia, Porcupine Press, 1989. Simons, H. C. (1962), «A positive program for laissez faire: Some proposals for a liberal economic policy», Economic Policy for a Free Society, Pittsburgh, Latin American Studies Association, publicado originalmente en 1934.

Singer, H. W. (1987), «Terms of trade and economic development», en John Eatwell, Murray Milgate y Peter Newman (editores), The New Palgrave. A Dictionary of Economics, Palgrave MacMillan.

(1950), «The distribution of gains between investing and borrowing countries», American Economic Review, vol. 40, Nashville, Tennessee, American Economic Association.

«The Economic Consequences of Mr. Churchill», en Essays in Persuasion, vol. Ix. The General Theory and After, parte II, Defense and Development, vol. XIV. «The Means to Prosperity», en Essays in Persuasion, vol. Ix. 
Vernengo, M. y E. Pérez Caldentey (2012a), «Heterodox Central Bankers: Eccles, Prebisch and financial reform», Network IDEAs Working Paper. (2012b), Prebisch's Dynamics, mimeo

Vernengo, M. y L. P. Rochon (2001), «Kaldor and Robinson on money and growth», European Journal of the History of Economic Thought, 8(1), pp. 75-103.

White, L. H. (2010), «Did Hayek and Robbins Deepen the Great Depression?», Universidad de Missouri, [en línea] 〈http://economics.sbs. ohio-state.edu/jmcb/jmcb/07056/07056.pdf . 